\title{
-Photogravure-
}

\section{Ultrastructure of Normal Rat Hepatocytes: Stereological Arrangement of the Organellae}

Shigeru Sato, Akiko Adachi, Yoshihiro Sasaki, Dai Wei and Yuichi Sugisaki

Central Institute for Electron Microscopic Researches, Nippon Medical School
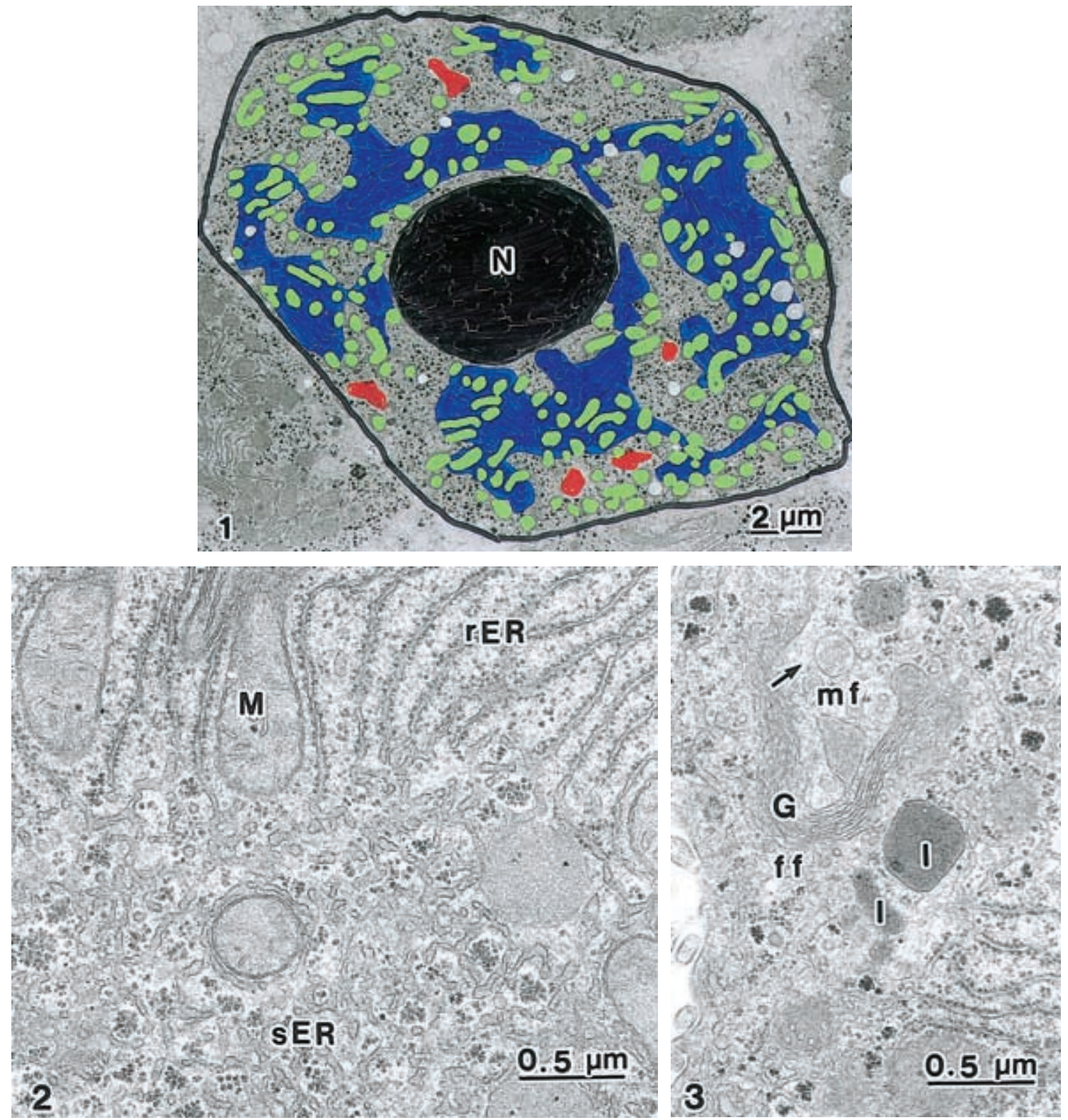

In the normal hepatocytes, the synthesis of albumin, fibrinogen and several other plasma proteins is a function of the rough endoplasmic reticulum ( $\mathrm{rER}$ ), while the cholesterol synthesis is a function of the smooth (s) ER. The Golgi complex is involved in the biliary secretion. In hepatic diseases, an increase in the hepatocyte's sER is often encountered. However, no baseline measure for the accurate estimation is available. Here, we illustrate a streological arrangement and measurement of the organellae in normal rat hepatocytes, that provide a basis for better understanding of the experimentally induced changes in hepatocytes and for improving the pathological hepatic diagnosis.

Journal Website (http://www.nms.ac.jp/jnms/) 

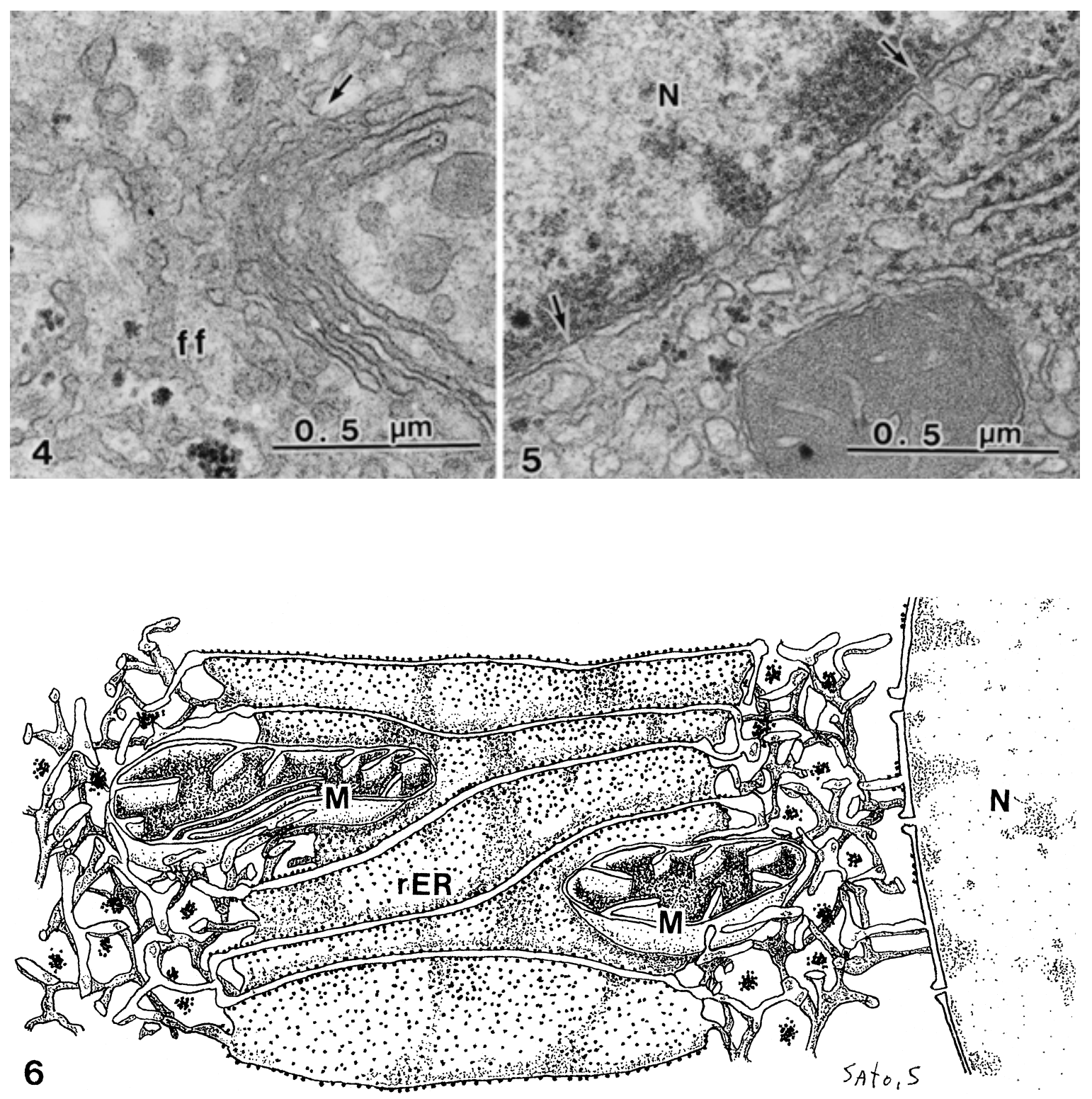

Fig. 1 Electron micrograph of a normal rat hepatocyte as analyzed by morphometry using the NIH image software shows nucleus $(\mathrm{N}), \mathrm{rER}$ area (blue painted;mean $=35 \pm 1.7 \%$ ), sER area (non-painted;mean $=60 \pm 1.8 \%$ ), Golgi complex area (red painted;mean $=4 \pm 1.7 \%$ ), mitochondria (green painted), and peroxisomes (white painted). The rER areas show a dendrite pattern. Many mitochondoria (green painted) are elongated, and spread between the sER and the rER.

Fig. 2 The sER presents as a network of branching tubules and contains the glycogen rossettes and a few lipid droplets. The rER lamellae plates contain free ribosomes and polyribosomes, and are communicated with the sER. M; mitochondria

Fig. 3 The Golgi complex is comprised of the parallel smooth surfaces of associated vesicles and contains secretory granules (arrow) and lysosomes (1). The ends of the elongated cisternae and the secretory granules are filled with an electron dense amorphous matrix including lipoproteins. The lysosome (peribiliary dense body) is observed in the forming face (ff) of the Golgi complex. mf; maturing face

Fig. 4 The Golgi cisternae at forming face (ff) are communicated with the sER (arrow).

Fig. 5 The nuclear envelope is communicated with the sER (arrows). N; Nucleus

Fig. 6 Diagram of the stereological arrangement of normal rat hepatocyte organellae showing that the sER (sER) is communicated with the rER (rER). N; nucleus M; mitochondria 